\title{
Vía intranasal: una alternativa para la administración de fármacos de acción central en equinos
}

\author{
Intranasal drug delivery: an alternative for the administration of central \\ acting drugs in horses
}

\author{
Ferreira Violeta (i] 1*, Velloso maría Inés (i] 2,4\#, Vita Mariángeles (i] 3, \\ LANDONI MARÍa Fabiana (10) 2* $^{*}$
}

1. Servicio de Medicina y Cirugía de Grandes Animales. Hospital Escuela. Facultad de Ciencias Veterinarias. Universidad Nacional de La Plata. 2. Cátedra de Farmacología General y Clínica. Facultad de Ciencias Veterinarias. Universidad Nacional de La Plata. ANPCyT. 3. Cátedra de Anatomía Comparada. Facultad de Ciencias Veterinarias. Universidad Nacional de La Plata. Argentina. 4. Becaria ANPCyT

* Correo electrónico de la autora de contacto: landoni@fcv.unlp.edu.ar \#igual colaboración

\begin{abstract}
Resumen
La vía intranasal es reconocida en medicina humana como una vía muy promisoria para la administración sistémica y cerebral de fármacos. Es una vía de administración indolora, incruenta, económica y práctica. Por sus características, es muy útil para la aplicación de fármacos en pacientes con alteraciones orales, con diarrea o que no cooperen. Además, es una potencial vía directa al sistema nervioso central (SNC). Su uso en medicina veterinaria es muy raro, salvo para la administración de tratamientos locales. En la especie equina, aun cuando no hay estudios completos y profundos sobre las características anatomofisiológicas de la cavidad nasal, su gran superficie e irrigación (área potencial de absorción) permiten inferir su viabilidad para la administración sistémica de fármacos. En el presente artículo se discuten las características de la cavidad nasal de los equinos en relación a las diversas vías de absorción sistémica y del transporte nariz-cerebro de fármacos, así como las potenciales aplicaciones de esta vía de administración.
\end{abstract}

\section{Palabras clave}

Vía intranasal, fármacos de acción central, pasaje nariz-cerebro, equinos

\begin{abstract}
The intranasal route is recognized as a very promising route for the systemic and cerebral administration of drugs in human medicine. It is a painless, non-invasive, economical, and practical administration route. Due to its characteristics, it is very useful for the application of drugs in non-cooperative patients, as well as those with oral alterations or diarrhoea or, being a potential direct route to the central nervous system (CNS). However, its use in veterinary medicine is very rare, except for the administration of local treatments. Even though there are no complete and thorough studies on the anatomophysiological characteristics of the nasal cavity in the equine species, its large surface area and irrigation and consequent potential absorption area suggest a promising alternative for the systemic administration of drugs. In the present article, the characteristics of the nasal cavity of horses in relation to the various routes of systemic absorption and nose-brain passage of drugs are discussed, as well as the potential applications of this route of administration.
\end{abstract}

\section{Key words}

Intranasal route, central acting drugs, nose-brain passage, horse

Fecha de recepción: 14/02/2019

Fecha de revisión: 12/04/2019

Fecha de aprobación: 02/05/2019
ANALECTA VeT 2019; Enero-Junio; 39(1):10-20

Impresa ISSN 03655 14-8 Electrónica ISSN 1514-2590

doi.org/10.24215/15142590eo33 


\section{Introducción}

La vía intranasal es reconocida en medicina humana como una vía muy promisoria para la administración sistémica y cerebral de fármacos (Falcone et al., 2014). Contrariamente, en medicina veterinaria es raramente utilizada; cuando se la utiliza, es solo para tratamientos locales con antihistamínicos o corticoides.

La vía intranasal en animales posee un gran potencial para la administración sistémica y cerebral de fármacos. En la especie equina, aun cuando no hay estudios completos y profundos sobre las características anatomofisiológicas de la cavidad nasal, su gran superficie e irrigación (área potencial de absorción) permiten inferir su viabilidad para la administración sistémica de fármacos.

Esta vía cuenta con ventajas que incrementan el interés en su estudio: es indolora, incruenta y práctica. Por sus características, es muy útil para la aplicación de fármacos en pacientes con alteracio ${ }^{\circ}$ nes orales, con diarrea o que no cooperen. Además, es una potencial vía directa al sistema nervioso central (SNC) (Tayebati et al., 2013).

\section{Anatomía de la cavidad nasal}

La cavidad nasal es el único sitio anatómico donde se expone el sistema nervioso de manera directa al medio ambiente. El aire y las sustancias inspiradas ingresan a la cavidad nasal a través de los ollares.

La cavidad nasal se divide, en un plano medio, en dos compartimentos especulares mediante un septum nasal, formado por la lámina perpendicular del hueso etmoides, el vómer y el cartílago hialino (Hare, 1982). La pared dorsal está formada por los huesos nasal y frontal, las paredes laterales por el maxilar, incisivo y parte perpendicular del palatino y la pared ventral por las apófisis palatinas de los huesos incisivos, maxilares y las partes horizontales de los huesos palatinos. La lámina cribosa del etmoides separa las cavidades nasal y craneal. En dirección a la cavidad craneal tiene una forma cóncava, generando una fosa donde se ubica el bulbo olfatorio (Hare, 1982). Cada mitad de la cavidad nasal está ocupada por los cornetes nasales (conchas o turbinetes), dorsal, medio y ventral, que son las estructuras encargadas del acondicionamiento del aire en su tránsito hacia los pulmones. Entre los cornetes quedan espacios denominados meatos. El meato nasal dorsal se encuentra entre el techo de la cavidad nasal y el cornete nasal dorsal. El meato nasal medio se ubica entre los cornetes dorsal y ventral. En este meato existe una abertura nasomaxilar que comunica con el seno maxilar. El meato nasal ventral, limitado por el cornete nasal ventral y el piso de la cavidad nasal, es el más largo y conduce hacia la nasofaringe. En el suelo del extremo rostral de este meato se ubica el orificio del órgano vomeronasal y el conducto incisivo. Por último, se encuentra el meato nasal común, situado entre los cornetes nasales y el tabique nasal (Fig. 1).

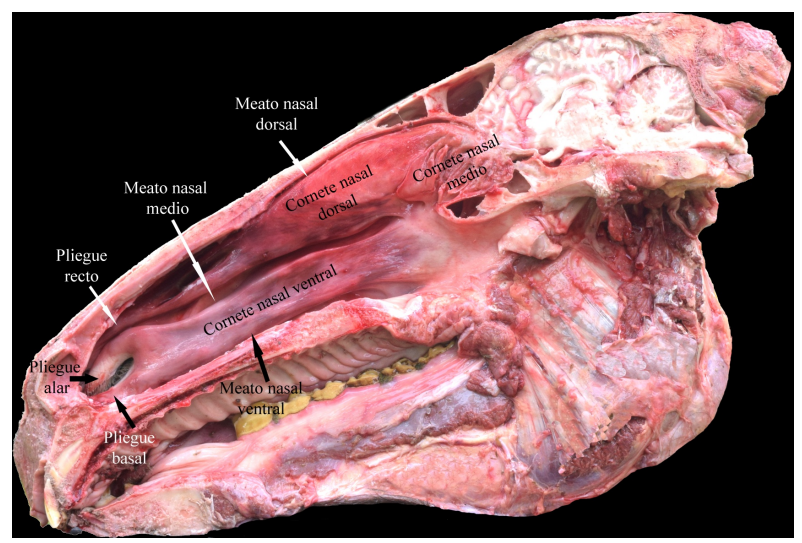

Figura 1. Imagen de una cabeza equina, señalando los sitios anatómicos de la cavidad nasal.

En la porción rostral de la cavidad nasal se encuentran pliegues que se prolongan desde los cornetes, desde el dorsal, el pliegue recto y desde el ventral los pliegues alar y basal.

La cavidad nasal está muy vascularizada; se encuentra irrigada por las arterias palatina mayor, esfenopalatina y ramas de la etmoidal. Además, se encuentra inervada por el nervio trigémino, cuyas ramas llevan información quimiosensorial y termosensorial desde las mucosas oral, ocular y nasal (Bourganis et al., 2018; Tayebati et al., 2013).

La mucosa de la porción rostral de la cavidad nasal o vestíbulo está cubierta por un epitelio plano estratificado no queratinizado que se continúa con un epitelio cilíndrico ciliado pseudoestratificado con células caliciformes de la porción respiratoria. El vestíbulo, en el cual se encuentra la desembocadura del conducto lagrimal, carece prácticamente de capacidad de absorción.

La mucosa de la porción respiratoria es de color rojizo a causa de la gran vascularización de su submucosa y contiene un rico plexo venoso que forma un tejido de tipo cavernoso en regiones como los pliegues que se extienden rostralmente a partir de los cornetes, la parte ventral del cornete nasal ventral y ventral del septum nasal.

La función primordial de la mucosa respiratoria es el calentamiento y la humidificación del aire inspirado. Normalmente su superficie está recubierta por el mucus segregado por las células caliciformes. El mucus, junto con las partículas de polvo que se le adhieren, se desplaza hacia la nasofaringe por acción de los cilios de las células de revestimiento epitelial, otorgándole una función protectora: el aclaramiento (clearance) ciliar (Gänge \& Schindowski, 2018; Lochhead \& Throne, 2014). 
La mucosa que cubre las partes caudales de los huesos etmoturbinados y las aéreas adyacentes del cornete nasal dorsal y septum nasal es gruesa y amarillenta y representa la región olfatoria de la cavidad nasal.

La mucosa olfatoria está formada por un epitelio y una lámina propia densa. El epitelio consta de 3 tipos de células: las epiteliales, llamadas también células de sostén o sustentaculares, las olfatorias o sensoriales y las basales (Lochhead \& Thorne, 2014) (Fig. 2).

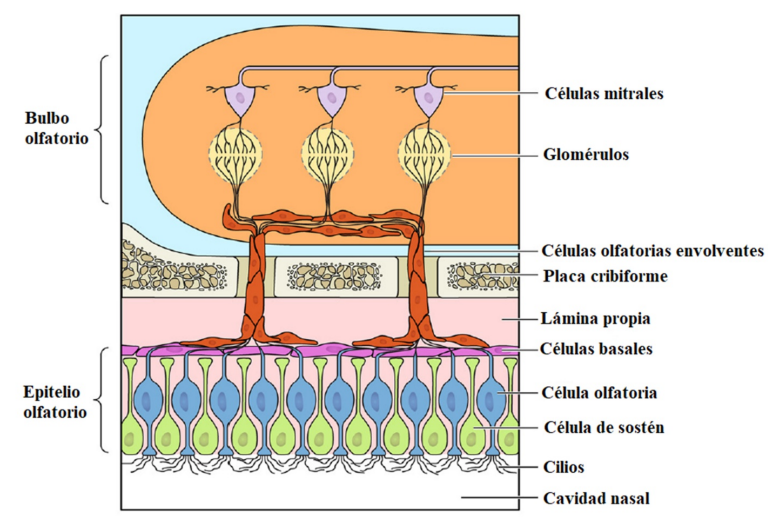

Figura 2. Representación esquemática del sistema olfatorio y su función como un portal para el pasaje de fármacos desde la cavidad nasal hasta el bulbo olfatorio, sorteando la barrera hematoencefálica. Figura adaptada de Dando et al., 2014.

Las células olfatorias o sensoriales (neuronas olfativas periféricas) son neuronas de tipo bipolar, con un cuerpo celular y una fibra nerviosa que emerge de cada extremo; una de ellas, la dendrita, es periférica, muy corta y de conducción sensitiva centrípeta y, la otra, el axón, es una prolongación central de conducción centrífuga.

Las células olfatorias tienen la función de recoger las impresiones olorosas y transmitir esta información sensorial desde el ambiente periférico hasta el SNC. Las finas prolongaciones constituidas por las dendritas ascienden hacia la parte superficial siguiendo las hendiduras que quedan entre las células de sostén y terminan en finísimas fibras llamadas cilios olfativos.

Por su parte, el axón va desde el epitelio hacia la lámina propia. Los axones en la lámina propia se encuentran protegidos por las células olfatorias envolventes (OEC, por sus siglas en inglés) (Lochhead \& Throne, 2014). Hay varias clases de OEC y están involucradas en la protección electrofisiológica de las células neuronales olfatorias (Gänge \& Schindowski, 2018). Los axones en la lámina propia se reúnen para formar haces de fibras nerviosas olfatorias que se extienden hasta el bulbo olfatorio, constituyendo, en conjunto, el nervio olfatorio.

En el bulbo, las fibras del nervio olfatorio terminan en una arborización libre y flexuosa. La terminación de cada fibra queda incluida en una formación redondeada llamada glomérulo olfatorio. Esta formación hace sinapsis con la arborización dendrítica de las células mitrales presentes en la zona interna del bulbo. Las células mitrales, por su parte opuesta, emiten los cilindroejes que avanzan hacia la región posterior del bulbo olfatorio para constituir la cinta olfativa, que se divide en 4 raíces. Cada una de ellas sigue un trayecto especial que la conecta a uno de los 4 centros corticales: calloso, hipocampo, temporal y orbitario, que representan los centros corticales del olfato. Desde la mucosa nasal las impresiones olfativas se trasladan a la corteza cerebral siguiendo este trayecto nervioso que en su conjunto constituye la vía olfativa (Dhuria et al., 2010).

Los nervios olfatorio y trigémino inervan la mucosa de la cavidad nasal y proporcionan una conexión directa con el SNC. El trigémino inerva principalmente el epitelio respiratorio y el vestíbulo de la cavidad nasal, aunque curiosamente una pequeña porción del nervio termina también en el epitelio olfatorio (Schaefer et al., 2002). También, establece conexión con el SNC, especialmente con la región caudal del cerebro, el tallo cerebral y la médula espinal, y transmite la información sensorial desde la mucosa nasal hasta estas regiones (Clerico et al., 2003; Gray, 1978).

El sistema olfatorio también está compuesto por órganos accesorios como el órgano vomeronasal. Este se encuentra en las dos fosas nasales y está conectado al bulbo olfatorio a través de un conducto. Su superficie está cubierta por epitelio olfatorio y células neuronales. Tiene una función importante en el comportamiento de las especies porque es capaz de detectar feromonas.

Todas estas características anatomofisiológicas le dan a la cavidad nasal atributos únicos que facilitan la llegada de los agentes terapéuticos directamente desde su mucosa hacia el SNC, eludiendo la barrera hematoencefálica.

\section{Transporte cavidad nasal-SNC}

El pasaje directo de agentes terapéuticos desde la cavidad nasal al SNC se propuso por primera vez y fue patentado en 1989 por William H. Frey II del Alzheimer's Research Center (Frey, 1991).

Posteriormente, numerosos informes han demostrado que los fármacos administrados por la vía intranasal acceden de manera directa al SNC aumentando su eficacia para el tratamiento de enfermedades y trastornos neurológicos (Dhanda et al., 2005; Frey, 2002; Hanson \& Frey, 2008).

Se ha demostrado que ciertos marcadores, como la aglutinina de germen de trigo conjugada con peroxidasa de rábano picante (WGA-HRP), son transportados a través de los axones del nervio olfatorio, alcanzando los bulbos olfatorios en el SNC (Balin et al., 1986). Estos hallazgos fueron posteriormente confirmados por otros estudios 
cuantitativos en los que se comparó la administración de WGA-HRP intranasal e intravenosa en ratas, siendo muy superior la llegada de WGAHRP a los bulbos olfatorios tras la administración intranasal (Thorne et al., 1995).

El conocimiento de las vías y los mecanismos de pasaje de fármacos desde la cavidad nasal hasta el SNC es fundamental para avanzar en el desarrollo de tratamientos intranasales de enfermedades y trastornos neurológicos, así como para la administración de fármacos de acción central, como los analgésicos opioides.

$\mathrm{Si}$ bien los mecanismos subyacentes del pasaje de fármacos desde la cavidad nasal al SNC no son del todo comprendidos, varias investigaciones demuestran la gran importancia de los nervios olfatorio y trigémino, que conectan la cavidad nasal con el cerebro y la médula espinal.

Se han reportado otras vías de pasaje, aunque menores, que involucran la vasculatura, líquido cefalorraquídeo (LCR) y sistema linfático. Estas vías actuarían amplificando el transporte de moléculas desde la cavidad nasal al SNC.

Es probable que la combinación de todas estas vías sea la responsable del pasaje total. La predominancia de cada una de ellas en el proceso dependerá de las características fisicoquímicas del fármaco, de la formulación del medicamento y del tipo de dispositivo de entrega utilizado (actuador nasal) (Dhuria et al., 2010).

\subsection{Vía del nervio olfatorio}

Los fármacos administrados por la vía intranasal pueden acceder rápidamente al SNC transportándose a lo largo de la vía neural del nervio olfatorio.

El nervio olfatorio es el componente principal de la conexión directa entre la cavidad nasal y el SNC. En este contexto, se ha reportado que fármacos coligados con marcadores fluorescentes son transportados a través de la placa cribiforme asociados a los nervios olfatorios (Jansson \& Bjork, 2002), alcanzando las concentraciones máximas en los bulbos olfatorios (Thorne et al., 2004). La correlación positiva entre las concentraciones en el epitelio olfatorio y en los bulbos olfatorios ha sido reportada (Dhuria et al., 2009).

Las vías olfatorias tienen su origen en la porción caudal de la cavidad nasal, en la región olfatoria, donde las neuronas receptoras olfatorias (ORN) están intercaladas entre las células de soporte (células sustentaculares), células sensoriales y células basales. Las ORNs median el sentido del olfato al transmitir información sensorial del entorno periférico al SNC (Clerico et al., 2003). La lámina propia contiene glándulas de Bowman, axones, vasos sanguíneos, vasos linfáticos y tejido conectivo. Las dendritas de las ORNs se extienden a la capa mucosa del epitelio olfatorio, mientras que los axones bipolares se extienden centralmente a través de la lámina propia y a través de perforaciones en la placa cribiforme del hueso etmoidal. Los axones de las ORNs pasan a través del espacio subaracnoideo, que contiene LCR, y terminan en las células mitrales de los bulbos olfatorios. A partir de ahí, las proyecciones neuronales se extienden a regiones cerebrales múltiples incluyendo el tracto olfatorio, núcleo olfatorio anterior, corteza piriforme, amígdala e hipotálamo (Buck, 2000).

Los mecanismos de transporte a lo largo de los nervios olfativos son de dos tipos, extracelulares e intracelulares (Fig 3).

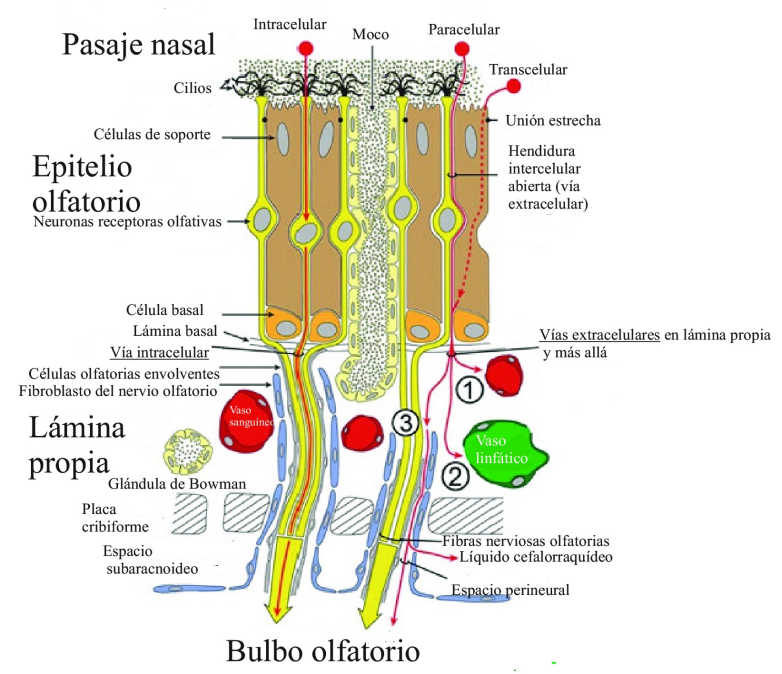

Figura 3. Representación esquemática de las vías de transporte de fármacos desde la cavidad nasal a la circulación sistémica y pasaje directo al SNC. (1) Absorción a través de los capilares olfatorios y entrada en la circulación sistémica. (2) Absorción a través de los vasos linfáticos de la región olfatoria que drenan en los linfonódulos profundos del cuello. (3) Difusión o convección extracelular en compartimentos asociados con haces de nervios olfativos y entrada en el compartimento craneal. Se muestra el transporte dentro del espacio perineural limitado por fibroblastos del nervio olfatorio. Sin embargo, existen otras posibilidades, por ejemplo, el transporte dentro del compartimento de la neurona olfatoria contenida por células envolventes, el transporte dentro de los espacios perivasculares de los vasos sanguíneos que atraviesan la lámina cribriforme junto con nervios olfativos (no se muestra) o el transporte dentro de los vasos linfáticos que atraviesan la lámina cribriforme con nervios olfativos (no se muestra). Se muestran las posibles vías para la distribución de sustancias desde el espacio perineural hacia el líquido cefalorraquídeo (LCR) del espacio subaracnoideo olfativo o hacia el bulbo olfativo. Adaptada de Lochhead \& Thorne, 2014.

Los mecanismos extracelulares de transporte se consideran los más importantes. Implican el pasaje rápido de moléculas desde las células del epitelio nasal hasta el SNC; se ha reportado que la administración intranasal de neurotropinas, neuropéptidos, citoquinas y carbamazepina conduce, 
en un lapso menor a los 10 minutos, a niveles detectables en los bulbos olfatorios y otras áreas del SNC (Frey, 2002).

El transporte extracelular involucra mecanismos de flujo masivo a través de los espacios (canales) existentes entre las neuronas y las células envolventes (Thorne \& Frey, 2001). Alternativamente, los fármacos también pueden ser propulsados a través de estos canales por los cambios estructurales que ocurren durante la despolarización y propagación axonal del potencial de acción en los axones adyacentes (Luzzati et al., 2004).

El mecanismo de transporte intracelular, si bien es importante, es probable que no sea la vía predominante de transporte de fármacos hacia el SNC. Este involucra la entrada de moléculas a las neuronas por difusión pasiva, endocitosis mediada por receptor y/o endocitosis adsorbente, seguida por transporte axonal lento (Banks et al., 2004; Hashizume et al., 2008; Nonaka et al., 2008; Ross et al., 2004; Thorne et al., 2004).

Este pasaje es mucho más lento que el extracelular, requiriéndose horas, hasta días, para la llegada del fármaco a los bulbos olfatorios y otras áreas cerebrales tras su administración intranasal (Baker \& Spencer, 1986; Broadwell \& Ballin, 1985; Kristensson \& Olsson, 1971). Este tipo de pasaje ha sido reportado para partículas de oro y sales de aluminio (Perl \& Good, 1987).

La mayoría de los estudios de administración intranasal de fármacos publicados hasta la fecha reporta una entrega rápida de fármaco, con altas concentraciones en el SNC y efectos clínicos inmediatos, consistente con el mecanismo extracelular de transporte (Banks et al., 2004; Charlton et al., 2008; Hanson \& Frey, 2008; Hashizume et al., 2008; Nonaka et al., 2008; Ross et al., 2004; Thorne et al., 2004).

\subsection{Vía del nervio trigémino}

La vía del nervio trigémino es un tipo de pasaje poco reportado. Sin embargo, en los últimos años su importancia como conexión entre la cavidad nasal y el SNC está siendo reconocida; ha sido reportada la distribución cerebral de fármacos administrados por la vía intranasal a zonas asociadas directamente a la vía del nervio trigémino, como tronco encefálico y cerebelo (Charlton et al., 2008; Banks et al., 2004).

El nervio trigémino inerva el epitelio respiratorio y olfativo de la cavidad nasal, entrando posteriormente al SNC a nivel del puente (Clerico et al., 2003; Gray, 1978). Curiosamente, una pequeña porción del nervio trigémino termina en los bulbos olfatorios (Cauna, 1982).

El transporte de fármacos administrados por la vía intranasal a lo largo de las vías del nervio trigémino fue demostrado por Thorne et al. (2004). Estos autores administraron factor de crecimiento similar a la insulina tipo I marcada con 125I (125I-IGF-I) por vía intranasal y observaron altos niveles de radiactividad en las ramas del nervio trigémino, el ganglio trigeminal, protuberancia y bulbos olfatorios, consistente con el transporte de la sustancia radioactiva a lo largo de los nervios trigémino y olfatorio (Thorne et al., 2004).

El nervio trigémino conduce información sensorial desde la cavidad nasal, la cavidad oral, los párpados y la córnea al SNC a través de sus tres ramas: el nervio oftálmico (V1), el maxilar (V2) y el mandibular (V3) (Gray, 1978). Las ramas de la porción oftálmica inervan la mucosa nasal dorsocraneal, mientras que las ramas de la porción maxilar inervan a las paredes laterales de la cavidad. La porción mandibular se extiende a la mandíbula inferior y dientes, sin entrada directa de neuronas a la cavidad nasal.

Las tres porciones del nervio trigémino se unen en el ganglio trigeminal y se extienden centralmente hasta su ingreso al cerebro a nivel de la protuberancia, terminando en los núcleos del nervio trigémino espinal en el tronco encefálico.

Una característica exclusiva del nervio trigémino es que ingresa al cerebro desde el epitelio respiratorio de las fosas nasales a través dos sitios: el foramen lacerum cerca de la protuberancia, y la lámina cribiforme, cerca de los bulbos olfatorios; de esta manera provee dos puntos de entrada para los fármacos administrados por vía intranasal, uno en el cerebro caudal y otro en el rostral (Dhuria et al., 2010).

También es probable que otros nervios que inervan la cara y la cabeza, como el nervio facial u otras estructuras sensoriales en la cavidad nasal, puedan proporcionar puntos de entrada de los agentes terapéuticos hacia el SNC.

\subsection{Vías vasculares}

Tradicionalmente, la vía de administración intranasal ha sido utilizada para el suministro de fármacos a la circulación sistémica a través de la absorción en los vasos sanguíneos de la mucosa nasal.

El epitelio de la mucosa olfatoria recibe sangre de pequeñas ramas de la arteria oftálmica, mientras que la mucosa respiratoria recibe sangre desde una arteria de gran calibre derivada de la maxilar. La densidad de los vasos sanguíneos es mucho mayor en la mucosa respiratoria que en la olfatoria, haciendo que la región respiratoria sea un sitio ideal para la absorción sistémica de fármacos (DeSesso, 1993).

La vasculatura de la región respiratoria posee una mezcla de endotelios continuos y fenestrados (Grevers \& Herrman, 1987) permitiendo que tanto moléculas pequeñas como grandes puedan acceder a la circulación sistémica después de la administración intranasal. La llegada de fármacos al SNC tras su llegada a la circulación sistémica y el transporte posterior a través de la barrera hematoencefálica es posible, especialmente para 
moleculas pequeñas y lipofílicas, que ingresan más fácilmente a la sangre y pueden cruzar dicha barrera.

Una vía de ingreso directo al SNC a través de los vasos de la mucosa nasal está relacionada con la transferencia de fármacos desde la circulación venosa nasal a la circulación arterial (carótida) y de ahí al cerebro y la médula espinal; este proceso ha sido denominado "transferencia de contracorriente" (Einer-Jensen \& Hunter, 2005).

Por otro lado, se ha reportado un segundo mecanismo de transporte directo al SNC que involucra los canales asociados con los vasos sanguíneos de la mucosa nasal, también llamados canales perivasculares. Los espacios perivasculares son limitados por la capa más externa de los vasos sanguíneos y la membrana basal del tejido circundante (Pollock et al., 1997) y actúan como sistema linfático para el cerebro; a través de ellos sustancias derivadas de las neuronas se eliminan desde el fluido intersticial cerebral a los vasos sanguíneos. Este tránsito ha sido demostrado a través de la utilización de sustancias radiomarcadas, tinta china y beta amiloide (Bradbury et al., 1981; Zhang et al., 1992).

El transporte perivascular es, a diferencia de la difusión, un mecanismo de flujo masivo (Groothuis et al., 2007) que obtiene su energía cinética de las pulsaciones (Rennels et al., 1985). La presencia de la denominada "bomba perivascular de transporte" explica la distribución rápida de los agentes terapéuticos a todo el cerebro (Hadaczek et al., 2006; Schley et al., 2005). De esta manera los fármacos administrados por la vía intranasal pueden ingresar a los espacios perivasculares de la cavidad llegando posteriormente al cerebro con una distribución generalizada.

\subsection{Vía del LCR - vasos linfáticos}

El drenaje del LCR incluye las vías que conectan el espacio subaracnoideo, los espacios perineurales que acompañan los nervios olfatorios y los linfáticos nasales; todas estas vías han demostrado proporcionar acceso y distribución dentro del SNC a los agentes terapéuticos administrados por vía intranasal. Si bien a la fecha no existen estudios en equinos, en otras especies animales, ovinos, lagomorfos y roedores, estas vías de drenaje representan aproximadamente $50 \%$ del clearance del LCR (Boulton et al., 1999; Bradbury et al., 1981; Cserr et al., 1992).

Varios estudios documentan que los marcadores inyectados en el LCR a nivel de los ventrículos cerebrales drenan desde el espacio subaracnoideo a la parte inferior de las bombillas olfativas llegando al sistema linfático nasal y linfonódulo cervical. Los fármacos administrados por la vía intranasal utilizan estas vías, moviéndose en dirección opuesta, para acceder y distribuirse dentro del SNC (Kida et al., 1993; Walter et al., 2006).

\section{Factores que afectan la absorción sisté- mica y el transporte de fármacos adminis- trados por la vía intranasal}

Como en todo proceso de absorción, independientemente del lugar en el que se lleve a cabo, el primer paso es el pasaje a través de la mucosa.

La absorción nasal de fármacos es afectada por el peso molecular, tamaño y pK del fármaco, así como el $\mathrm{pH}$ de la formulación y el volumen administrado (Agarwal \& Mishra, 1999; Fisher et al., 1987). Sin embargo, el factor que mejor correlaciona con la absorción intranasal es el peso molecular; las moléculas pequeñas y sin carga son las de mayor velocidad de pasaje. Se reporta que el peso molecular máximo de una molécula para atravesar la mucosa nasal es de 1000 daltons (Wheatley et al., 1988).

La forma de la molécula es también importante. Las moléculas rectangulares o cuadradas tienen menor biodisponibilidad que las de forma circular (McMartin et al., 1987). Adicionalmente, es importante recordar que las partículas deben tener un tamaño superior a los $10 \mathrm{~nm}$ para evitar el depósito directo en el pulmón (Jones et al., 1997).

La hidrofilicidad es otro factor que reduce la biodisponibilidad del fármaco (Corbo et al., 1989), al igual que el pH de la cavidad nasal y el $\mathrm{pK}$ del fármaco a administrar. Es importante remarcar que butorfanol es un ácido débil, por lo que se encuentra no ionizado en medios ácidos. En este contexto, es importante mantener el $\mathrm{pH}$ de las formulaciones en el rango de 4,5 a 6,5 para evitar la irritación nasal (Romeo et al., 1998).

Dentro de los factores locales que pueden alterar la absorción de los fármacos administrados por vía intranasal, se incluyen el clearance mucociliar y el metabolismo enzimático local.

El clearance mucociliar es uno de los factores locales con mayor influencia en la biodisponibilidad de los fármacos administrados por vía intranasal, especialmente aquellos que atraviesan las membranas lentamente (moléculas de gran tamaño o de baja liposolubilidad) o son administrados en forma de polvos. El tiempo de barrido mucociliar nasal en equinos no ha sido reportada; en humanos es de entre 15 y 30 minutos (Soane et al., 1999). Una estrategia para mejorar la biodisponibilidad de fármacos con malos perfiles fisicoquímicos para administración intranasal es realizar la administración en el límite más externo del vestíbulo nasal (Kublik \& Vidgern, 1998).

En relación con la actividad enzimática en la mucosa nasal como factor limitante de la administración de fármacos, es solo aplicable a fármacos con estructura peptídica y a proteínas. Esto es consecuencia de que la actividad enzimática a este nivel se limita a exo- y endopeptidasas (Lee \& Yamamoto, 1989; Morimoto et al., 1995). 


\section{Aplicaciones clínicas de la vía intranasal}

A diferencia de lo que sucede en medicina veterinaria, la vía intranasal es ampliamente utilizada en medicina humana.

Esta vía se utiliza para la aplicación de una amplia gama de vacunas, incluyendo las vacunas contra difteria (Alpar et al., 2001), tétanos (Alpar et al., 2001), influenza (Singh et al., 2001), cólera (Yuki et al., 2001) y virus de inmunodeficiencia humana (VIH) (Velin \& Kraehenbuhl, 2000). Su uso también ha sido reportado para la administración de péptidos y proteínas como insulina (Benedict et al., 2011), calcitonina (Lochhead \& Throne, 2012), hormona del crecimiento (Hedin et al., 1993) y desmopresina (Lopes et al., 2008), entre otros. Su aplicación para lograr una vía directa al SNC (nose to brain delivery) está siendo ampliamente desarrollada para antiepilépticos, como carbamazepina (Serralheiro et al., 2014) opioides, como fentanilo, alfentanilo, butorfanol, oxicodona (Grassin-Delyle et al., 2012) y benzodiazepinas, como diazepam (Sharma et al., 2015).

A manera de ejemplo para demostrar la potencialidad de la vía intranasal, en la Tabla 1 se presentan algunas de las formulaciones comerciales en el mercado farmacéutico humano (GrassinDelyle et al., 2012).

\begin{tabular}{|c|c|c|}
\hline $\begin{array}{l}\text { PRINCIPIO } \\
\text { ACTIVO }\end{array}$ & $\begin{array}{l}\text { NOMBRE } \\
\text { COMERCIAL }\end{array}$ & INDICACIONES \\
\hline Buserelina & Suprefact nasal® & Cáncer de próstata \\
\hline Nafarelina & Synarel $\mathbb{Q}$ & Endometriosis \\
\hline Desmopresina & $\operatorname{Minirin}(\mathbb{B})$ & $\begin{array}{l}\text { Prevención y control de la polidipsia, } \\
\text { poliuria y deshidratación en pacientes con } \\
\text { diabetes insípida }\end{array}$ \\
\hline Calcitonina & Miacalcin $\mathbb{Q}$ & Osteoporosis post-menopáusica \\
\hline Dihidroergotamina & Diergo-spray@ & Migraña \\
\hline Sumatriptan & $\operatorname{Imigran}(\mathbb{B}$ & Migraña \\
\hline Butorfanol & Stadol NS@ & Dolor \\
\hline Fentanilo & Instany $1 \otimes$, ecFent $₫$ & Dolor \\
\hline Estradiol & Aerodiol $\mathbb{B}$ & Terapia de reemplazo hormonal \\
\hline Nicotina & Nicotrol NS® & Control fumadores \\
\hline Oxitocina & Syntocinon $\otimes$ & $\begin{array}{l}\text { Inducción al parto y estimulación de la } \\
\text { lactancia }\end{array}$ \\
\hline Cianocobalamina & Nascobal $\mathbb{B}$ & Deficiencia de Vit B12 \\
\hline $\begin{array}{l}\text { Vacuna de } \\
\text { Influenza }\end{array}$ & FluMist $₫$ & Prevención de gripe N1H1 \\
\hline
\end{tabular}

Tabla 1. Formulaciones comerciales de administración intranasal encontradas en el mercado farmacéutico humano (Grassin-Delyle et al., 2012).

Como se mencionó previamente, el uso de la vía intranasal en equinos es muy limitado. Desde hace unos años nuestro grupo de investigación se encuentra abocado al estudio de esta vía de administración en equinos, habiéndose desarrollado diversas formulaciones experimentales de butorfanol para administración intranasal.
A la fecha hemos reportado que la administración intranasal es tolerada por los equinos; no se observan muestras de rechazo ni irritación tras la administración de 3 disparos consecutivos de $200 \mu \mathrm{l}$ de solución fisiológica o formulación experimental (Ferreira et al., 2015). Asimismo, hemos reportado una biodisponibilidad cercana al 60 \% para una formulación intranasal de butorfanol desarrollada en nuestro laboratorio (Ferreira et al., 2018).

\section{Conclusiones}

En conclusión, la vía de administración intranasal es una alternativa válida de la vía intravenosa para la administración de fármacos en equinos. Además de sus características de practicidad, ausencia de dolor y abrasión de epitelios, la presencia de un transporte directo al SNC, la convierte en una importante herramienta terapéutica para la administración de fármacos de acción central.

\section{Conflicto de intereses}

Los autores declaran que no existe conflicto de intereses, incluyendo entre estos últimos las relaciones financieras, personales o de otro tipo con otras personas u organizaciones que pudieran influir de manera inapropiada en el trabajo.

\section{Bibliografía}

Agarwal V, Mishra B. 1999. Recent trends in drug delivery systems: intranasal drug delivery. Indian Journal of Experimental Biology. 37(1):6-16.

Alpar HO, Eyles JE, Williamson ED, Somavarapu S. 2001. Intranasal vaccination against plague, tetanus and diphtheria. Advanced Drug Delivery Reviews. 51(1-3):173-201.

Baker H, Spencer RF. 1986. Transneuronal transport of peroxidase-conjugated wheat germ agglutinin (WGA-HRP) from the olfactory epithelium to the brain of the adult rat. Experimental Brain Research. 63:461-73.

doi: 10.1007/BFo0237470

Balin BJ, Broadwell RD, Salcman M, el-Kalliny M. 1986. Avenues for entry of peripherally administered protein to the central nervous system in mouse, rat, and squirrel monkey. The Journal of Comparative Neurology. 251(2):26080. doi: 10.1002/cne.902510209

Banks WA, During MJ, Niehoff ML. 2004. Brain uptake of the glucagon-like peptide-1 antagonist 
exendin(9-39) after intranasal administration. Journal of Pharmacology and Experimental Therapeutics. 309 (2):469-75.

doi: 10.1124/jpet.103.063222

Benedict C, Frey WH 2nd, Schiöth, HB, Schultes B, Born J, Hallschmid M. 2011. Intranasal insulin as a therapeutic option in the treatment of cognitive impairments. Experimental Gerontology. 46(2-3): 112-5. doi: 10.1016/j.exger.2010.08.026

Boulton M, Flessner M, Armstrong D, Mohamed R, Hay J, Johnston M. 1999. Contribution of extracranial lymphatics and arachnoid villi to the clearance of a CSF tracer in the rat. American Journal of Physiology. 276(3):R818-R23.

doi: 10.1152/ajpregu.1999.276.3.R818

Bourganis V, Kammona O, Alexopoulos A, Kiparissides C. 2018. Recent advances in carrier mediated nose-to-brain delivery of pharmaceutics. European Journal of Pharmaceutics and Biopharmaceutics. 128:337-62.

doi: 10.1016/j.ejpb.2018.05.009

Bradbury MW, Cserr HF, Westrop RJ. 1981. Drainage of cerebral interstitial fluid into deep cervical lymph of the rabbit. American Journal of Physiology. 240(4):F329-F36.

doi: 10.1152/ajprenal.1981.240.4.F329

Broadwell RD, Balin BJ. 1985. Endocytic and exocytic pathways of the neuronal secretory process and trans synaptic transfer of wheat germ agglutinin-horseradish peroxidase in vivo. The Journal of Comparative Neurology. 242(4):63250. doi: 10.1002/cne.902420410

Buck LB. 200o. The chemical senses. En: Kandel ER, Schwartz JH, Jessell TM, editors. Principles of neural science. 4th edition. New York, McGrawHill Companies. pp. 625-52.

Cauna N. 1982. Blood and nerve supply of the nasal lining. En: Proctor DF, Andersen I, editors. The nose: Upper airway physiology and the atmospheric environment. Amsterdam: Elsevier Biomedical Press. pp. 45-69.

Charlton ST, Whetstone J, Fayinka ST, Read KD, Illum L, Davis SS. 2008. Evaluation of direct transport pathways of glycine receptor antagonists and an angiotensin antagonist from the nasal cavity to the central nervous system in the rat model. Pharmaceutical Research. 25(7):1531-43. doi: 10.1007/s11095-008-9550-2

Clerico DM, To WC, Lanza DC. 2003. Anatomy of the human nasal passages. En: Doty RL, editor. Handbook of olfaction and gustation. 2nd edition. New York: Marcel Dekker. Inc. pp. 1-16.
Corbo DC, Huang YC, Chien, YW. 1989. Nasal delivery of progestational steroids in ovariectomized rabbits. II. Effect of penetrant hydrophilicity. International Journal of Pharmaceutics. 50(3):253-60.

doi: 10.1016/0378-5173(89)90128-2

Cserr HF, Harling-Berg CJ, Knopf PM. 1992. Drainage of brain extracellular fluid into blood and deep cervical lymph and its immunological significance. Brain Pathology. 2 (4):269-76.

doi: 10.1111/j.1750-3639.1992.tboo703.x

Dando SJ, Mackay-Sim A, Norton R, Currie BJ, St John JAS, Ekberg, JA, Batzloff M, Ulett GC, Beacham IR. 2014. Pathogens penetrating the central nervous system: infection pathways and the cellular and molecular mechanisms of invasion. Clinical Microbiology Reviews. 27(4): 691-726. doi: 10.1128/CMR.00118-13

DeSesso JM. 1993. The relevance to humans of animal models for inhalation studies of cancer in the nose and upper airways. Qual Assur. 2(3):21331.

Dhanda DS, Frey WH 2nd, Leopold D, Kompella UB. 2005. Approaches for drug deposition in the human olfactory epithelium. Journal of Drug Delivery Science and Technology. 5:64-72.

Dhuria SV, Hanson LR, Frey WH 2nd. 2009. Novel vasoconstrictor formulation to enhance intranasal targeting of neuropeptide therapeutics to the central nervous system. Journal of Pharmacology and Experimental Therapeutics. 328(1):312-20. doi: 10.1124/jpet.108.145565

Dhuria SV, Hanson LR, Frey WH 2nd. 2010. Intranasal delivery to the central nervous system: mechanisms and experimental considerations. Journal of Pharmaceutical Sciences. 99(4):165473. doi: 10.1002/jps.21924

Einer-Jensen N, Hunter RHF. 2005. Countercurrent transfer in reproductive biology. Society for Reproduction and Fertility. 129(1):9-18.

doi: 10.1530/rep.1.00278.

Falcone JA, Salameh, TS, Yi X, Cordy BJ, Mortell WG, Kabanov AV, Banks WA. 2014. Intranasal administration as a route for drug delivery to the brain: Evidence for a unique pathway for albumin. Journal of Pharmacology and Experimental Therapeutics. 351(1):54-60.

doi: 10.1124/jpet.114.216705

Ferreira V, Teme Centurion O, Monina M, Landoni, MF. 2015. Evaluación de la vía intranasal para la administración de opioides en equinos. V Jornadas de Jóvenes Investigadores "Ciencia y Sociedad". Buenos Aires. Invet 17(1):63. 
Ferreira V, Zapata G, Landoni MF. 2018. Disposición plasmática del butorfanol tras su administración intranasal e intravenosa en equinos. VIII Jornadas de Jóvenes Investigadores. Buenos Aires. Invet 20(1):120.

Fisher AN, Brown K, Davis SS, Parr GD, Smith DA. 1987. The effect of molecular size on the nasal absorption of water-soluble compounds in the albino rat. Journal of Pharmacy and Pharmacology. 39(5):357-62.

doi: 10.1111/j.2042-7158.1987.tbo3398.x

Frey WH II. 1991. En: WPTO, editor. Neurologic agents for nasal administration to the brain. US: Chiron Corporation.

Frey WH II. 2002. Bypassing the blood-brain barrier to delivery therapeutic agents to the brain and spinal cord. Journal of Drug Delivery Science and Technology. 2:46-9.

Gänger S, Schindowski K. 2018. Tailoring formulations for intranasal nose-to-brain delivery: a review on architecture, physicochemical characteristics and mucociliary clearance of the nasal olfactory mucosa. Pharmaceutics. 10(3):11644. doi: 10.3390/pharmaceutics10030116

Grassin-Delyle S, Buenestado A, Naline, E, Faisy C, Blouquit-Laye S, Couderc LJ, Le Guen M, Fischler M, Devillier P. 2012. Intranasal drug delivery: an efficient and non-invasive route for systemic administration: focus on opioids. Pharmacology \& Therapeutics. 134(3):366-79.

doi: 10.1016/j.pharmthera.2012.03.003

Gray H. 1978. Gray's anatomy. 15th revised edition (Classic Collectors edition). New York, Bounty Books.

Grevers G, Herrmann U. 1987. Fenestrated endothelia in vessels of the nasal mucosa. An electron-microscopic study in the rabbit. Archives of Otorhinolaryngology. 244(1):55-60.

Groothuis DR, Vavra MW, Schlageter KE, Kang EW, Itskovich AC, Hertzler S, Allen CV, Lipton HL. 2007. Efflux of drugs and solutes from brain: The interactive roles of diffusional transcapillary transport, bulk flow and capillary transporters. Journal of Cerebral Blood Flow and Metabolism. 27(1):43-56. doi: 10.1038/sj.jcbfm.9600315

Hadaczek P, Yamashita Y, Mirek H, Tamas L, Bohn MC, Noble C, Park JW, Bankiewicz K. 2006. The "perivascular pump" driven by arterial pulsation is a powerful mechanism for the distribution of therapeutic molecules within the brain. Molecular Therapy. 14(1):69-78.

doi: 10.1016/j.ymthe.2006.02.018
Hanson LR, Frey WH 2nd. 2008. Intranasal delivery bypasses the blood-brain barrier to target therapeutic agents to the central nervous system and treat neurodegenerative disease. BMC Neuroscience. 9:S5.

doi: 10.1186/1471-2202-9-S3-S5

Hare WCD. 1982. Capítulo 19: Sistema respiratorio de los equinos. En: Getty R, Sisson S, Grossman JD. Anatomía de los animales domésticos. Tomo I. 5 ta edición. Barcelona, Salvat Editores S.A, pp. 557-72.

Hashizume R, Ozawa T, Gryaznov SM, Bollen AW, Lamborn KR, Frey WH 2nd, Deen DF. 2008. New therapeutic approach for brain tumors: Intranasal delivery of telomerase inhibitor GRN163. NeuroOncology. 10(2):112-20.

doi: 10.1215/15228517-2007-052

Hedin L, Olsson B, Diczfalusy M, Flyg C, Petersson AS, Rosberg S, Albertsson-Wikland K. 1993. Intranasal administration of human growth hormone $(\mathrm{hGH})$ in combination with a membrane permeation enhancer in patients with $\mathrm{GH}$ deficiency: a pharmacokinetic study. The Journal of Clinical Endocrinology \& Metabolism. 76(4): 962-7. doi: 10.1210/jcem.76.4.8473411

Jansson B, Bjork E. 2002. Visualization of in vivo olfactory uptake and transfer using fluorescein dextran. Journal of Drug Targeting. 10(5):379-86 doi: 10.1080/1061186021000001823

Jones D, Woolfson AD, Brown AF. 1997. Textural, viscoelastic and mucoadhesive properties of pharmaceutical gels composed of cellulose polymers. International Journal of Pharmaceutics. 151(2):223-33.

doi: 10.1016/So378-5173(97)04904-1

Kida S, Pantazis A, Weller RO. 1993. CSF drains directly from the subarachnoid space into nasal lymphatics in the rat. Anatomy, histology and immunological significance. Neuropathology and Applied Neurobiology. 19(6):480-8.

doi: 10.1111/j.1365-2990.1993.tboo476.x

Kristensson K, Olsson Y. 1971. Uptake of exogenous proteins in mouse olfactory cells. Acta Neuropathologica. 19(2):145-54.

Kublik H, Vidgren MT. 1998. Nasal delivery systems and their effect on deposition and absorption. Advanced Drug Delivery Reviews. 29(1-2):157-77.

doi: 10.1016/So169-409X(97)00067-7

Lee VH, Yamamoto A. 1989. Penetration and enzymatic barriers to peptide and protein absorption. Advanced Drug Delivery Reviews. 4(2): 171-207. doi: 10.1016/0169-409X(89)90018-5 
Lochhead JJ, Thorne RG. 2012. Intranasal delivery of biologics to the central nervous system. Advanced Drug Delivery Reviews. 64(7):614-28. doi: 10.1016/j.addr.2011.11.002

Lochhead JJ, Thorne RG. 2014. Capítulo 14: intranasal drug delivery to the brain. En: Hammarlund-Udenaes M, Lange ECM, Thorne RG \& Editors Drug Delivery to the Brain: Physiological Concepts, Methodologies and Approaches. 1st edition. New York. aapspress. Springer. pp. 40132.

Lopes T, Dias JS, Marcelino J, Varela J, Ribeiro S, Dias J. 2008. An assessment of the clinical efficacy of intranasal desmopressin spray in the treatment of renal colic. BJU International. 87(4):322-5.

doi: 10.1046/j.1464-410x.2001.00068.x

Luzzati V, Benoit E, Charpentier G, Vachette P. 2004. X-ray scattering study of pike olfactory nerve: Elastic, thermodynamic and physiological properties of the axonal membrane. Journal of Molecular Biology. 343(1):199-212.

doi: 10.1016/j.jmb.2004.08.029

McMartin C, Hutchinson LE, Hyde R, Peters GE. 1987. Analysis of structural requirements for the absorption of drugs and macromolecules from the nasal cavity. Journal of Pharmaceutical Sciences. 76(7):535-40. doi: 10.1002/jps.2600760709

Morimoto K, Miyazaki M, Kakemi M. 1995. Effects of proteolytic enzyme inhibitors on nasal absorption of salmon calcitonin in rats. International Journal of Pharmaceutics. 113(1):1-8. doi: 10.1016/0378-5173(94)00158-2

Nonaka N, Farr SA, Kageyama H, Shioda S, Banks WA. 2008. Delivery of galanin-like peptide to the brain: Targeting with intranasal delivery and cyclodextrins. Journal of Pharmacology and Experimental Therapeutics. 325(2):513-19.

doi: 10.1124/jpet.107.132381

Perl PD, Good PF. 1987. Uptake of aluminium into central system along nasal-olfactory pathways. The Lancet. 329(8540):1028.

doi: 10.1016/So140-6736(87)92288-4

Pollock H, Hutchings M, Weller RO, Zhang ET. 1997. Perivascular spaces in the basal ganglia of the human brain: Their relationship to lacunes. Journal of Anatomy. 191:337-46.

doi: 10.1046/j.1469-7580.1997.19130337.x

Rennels ML, Gregory TF, Blaumanis OR, Fujimoto K, Grady PA. 1985. Evidence for a 'paravascular' fluid circulation in the mammalian central nervous system, provided by the rapid distribution of tracer protein throughout the brain from the subarachnoid space. Brain Research. 326(1):47-63. doi: 10.1016/0006-8993(85)91383-6
Romeo VD, deMeireles J, Sileno AP, Pimplaskar, HK, Behl CR. 1998. Effects of physicochemical properties and other factors on systemic nasal drug delivery. Advanced Drug Delivery Reviews. 29(1-2):89-116.

doi: 10.1016/So169-409X(97)ooo63-X

Ross TM, Martinez PM, Renner JC, Thorne RG, Hanson LR, Frey WH 2nd. 2004. Intranasal administration of interferon beta bypasses the bloodbrain barrier to target the central nervous system and cervical lymph nodes: A non-invasive treatment strategy for multiple sclerosis. Journal of Neuroimmunology. 151(1-2):66-77.

doi: 10.1016/j.jneuroim.2004.02.011

Schaefer ML, Böttger B, Silver WL, Finger TE. 2002. Trigeminal collaterals in the nasal epithelium and olfactory bulb: A potential route for direct modulation of olfactory information by trigeminal stimuli. Journal of Comparative Neurology. 444(3):221-6. doi: 10.1002/cne.10143

Schley D, Carare-Nnadi R, Please CP, Perry VH, Weller RO. 2005. Mechanisms to explain the reverse perivascular transport of solutes out of the brain. Journal of Theoretical Biology. 238(4):96274. doi: 10.1016/j.jtbi.2005.07.005

Serralheiro A, Alves G, Fortuna A, Falcão A. 2014. Intranasal administration of carbamazepine to mice: a direct delivery pathway for brain targeting. European Journal of Pharmaceutical Sciences. 60:32-9. doi: 10.1016/j.ejps.2014.04.019

Sharma S, Lohan S, Murthy, RSR. 2014. Formulation and characterization of intranasal mucoadhesive nanoparticulates and thermoreversible gel of levodopa for brain delivery. Drug Development and Industrial Pharmacy. 40(7): 869-78. doi: 10.3109/03639045.2013.789051

Singh M, Vajdy M, Gardner J, Briones M, O’Hagan D. 2001. Mucosal immunization with HIV-1 gag DNA on cationic microparticles prolongs gene expression and enhances local and systemic immunity. Vaccine. 20(3-4):594-602.

doi: 10.1016/So264-410X(01)00321-8

Soane RJ, Frier M, Perkins AC, Jones NS, Davis SS, Illum L. 1999. Evaluation of the clearance characteristics of bioadhesive systems in humans. International Journal of Pharmaceutics. 178(1):5565. doi: 10.1016/So378-5173(98)00367-6

Tayebati SK, Nwankwo IE, Amenta F. 2013. Intranasal drug delivery to the central nervous system: present status and future outlook. Current Pharmaceutical Design. 19(3):510-26.

doi: $10.2174 / 1381612811306030510$

Thorne RG, Emory CR, Ala TA, Frey WH 2nd. 1995. Quantitative analysis of the olfactory 
pathway for drug delivery to the brain. Brain Research. 692(1-2):278-82.

doi: 10.1016/0006-8993(95)oo637-6

Thorne RG, Pronk GJ, Padmanabhan V, Frey WH 2nd. 2004. Delivery of insulin-like growth factor-I to the rat brain and spinal cord along olfactory and trigeminal pathways following intranasal administration. Neuroscience. 127(2):481-96.

doi: 10.1016/j.neuroscience.2004.05.029

Thorne RG, Frey WH 2nd. 2001. Delivery of neurotrophic factors to the central nervous system: Pharmacokinetic considerations. Clinical Pharmacokinetics. 40(12):907-46.

doi: 10.2165/00003088-200140120-00003

Velin D, Kraehenbuhl JP. 200o. Delivery systems and adjuvants for vaccination against HIV. Pathobiology EXS. 89:227-37.

Walter BA, Valera VA, Takahashi S, Matsuno K, Ushiki T. 2006. Evidence of antibody production in the rat cervical lymph nodes after antigen administration into the cerebrospinal fluid.
Archives of Histology and Cytology. 69(1):37-47. doi: 10.1679/aohc.69.37

Wheatley MA, Dent J, Wheeldon EB, Smith PL. 1988. Nasal drug delivery: An in vitro characterization of transepithelial electrical properties and fluxes in the presence or absence of enhancers. Journal of Controlled Release. 8(2): 167-77. doi: 10.1016/0168-3659(88)90043-0

Yuki Y, Byun Y, Fujita M, Izutani W, Suzuki T, Udaka S, Fujihashi K, McGhee JR, Kiyono H. 2001. Production of a recombinant hybrid molecule of cholera toxin-B-subunit and proteolipid-protein-peptide for the treatment of experimental encephalomyelitis. Biotechnology and Bioengineering. 74(1):62-9.

doi: 10.1002/bit.1095

Zhang ET, Richards HK, Kida S, Weller RO. 1992. Directional and compartmentalised drainage of interstitial fluid and cerebrospinal fluid from the rat brain. Acta Neuropathologica. 83(3):233-9. 